\title{
BRANCHINGS AND PARTITIONS
}

\section{CARLITZ ${ }^{1}$ AND RICHARD P. STANLEY ${ }^{2}$}

ABSTRACT. A generating function is obtained for the number of partitions corresponding to a complete branching on a nonincreasing sequence of $n$ integers. Complete branchings are shown to be related to certain types of plane partitions.

Given a sequence $k_{1} \geq k_{2} \geq k_{3} \geq \cdots \geq k_{n}$ of integers, a branching is a sequence of integers $k_{1}^{\prime}, k_{2}^{\prime}, \ldots, k_{n-1}^{\prime}$ such that $k_{i} \geq k_{i}^{\prime} \geq k_{i+1}(i=1,2$, $\ldots, n-1)$. Successively branching $n-1$ times, one obtains a single integer. Such a sequence of $n-1$ successive branchings is called a complete branch. ing. S. Gelbart [3] proposed as a Monthly problem that the number of distinct complete branchings of the sequence $k_{1}, k_{2}, \ldots, k_{n}$ is equal to

$$
\prod_{1 \leq i<j \leq n}\left[\left(k_{i}-k_{j}+j-i\right) /(j-i)\right] .
$$

A complete branching may be indicated by the triangular array

$$
\begin{aligned}
& k_{1} k_{2} k_{3} \quad \cdots \quad k_{n} \\
& k_{1}^{\prime} \quad k_{2}^{\prime} \quad \cdots \quad k_{n-1}^{\prime} \\
& T_{n}: \quad k_{1}^{\prime \prime} \quad \ldots k_{n-2}^{\prime \prime} \\
& k_{1}^{(n-1)}
\end{aligned}
$$

where $k_{i}^{(O)}=k_{i}$ and

$$
k_{i}^{(j)} \geq k_{i}^{(j+1)} \geq k_{i+1}^{(j)} \quad(i=1,2, \ldots, n-j-1) .
$$

For a given integer $m$ and fixed sequence $k_{1} \geq k_{2} \geq \cdots \geq k_{n}$, let $P_{n}(m)$ $=P\left(m ; k_{1}, \ldots, k_{n}\right)$ denote the number of arrays $T_{n}$ satisfying (2) and whose entries sum to $m$, i.e.,

$$
m=\sum_{j=0}^{n-1} \sum_{i=1}^{n-j} k_{i}^{(j)} .
$$

Received by the editors October 27, 1973 and, in revised form, August 10, 1974 . AMS (MOS) subject classifications (1970). Primary 05A15, 10A45. tion.

Key words and phrases. Branching, partition, plane partition, generating func-

1 Supported in part by NSF grant GP-37924.

2 Supported in part by NSF grant GP-36739. 
We shall be interested in evaluating the generating function

$$
N\left(k_{1}, \ldots, k_{n} ; x\right)=N(x)=\sum_{m=-\infty}^{\infty} P_{n}(m) x^{m} .
$$

Note that the sum (3) is actually finite.

There is no loss of generality in supposing that the $k_{i}$ 's are all positive, for this amounts to adding some constant $c$ to each entry of $T_{n}$ and therefore multiplying (3) by $x^{c^{(n+1)}} 2$. Assuming therefore that each $k_{i}>0$, rewrite $T_{n}$ as

$$
\begin{aligned}
& k_{1} \quad k_{1}^{\prime} \quad k_{1}^{\prime \prime} \cdots k_{1}^{(n-1)} \\
& k_{2} k_{2}^{\prime} \cdots k_{2}^{(n-2)} \\
& \text {.. } \\
& k_{n}
\end{aligned}
$$

The $i$ th row satisfies $k_{i} \geq k_{i}^{\prime} \geq \cdots \geq k_{i}^{(n-i)}>0$ and therefore may be regarded as a partition of $k_{i}+k_{i}^{\prime}+\cdots+k_{i}^{(n-i)}$. Take the conjugate partition [4, p. 274] of each row and left justify, giving a new array

$$
\begin{array}{lll}
j_{1} & & j_{1}^{\prime} \cdots \\
j_{2} & & j_{2}^{\prime} \cdots \\
& \ldots & \\
& & \\
j_{n} & & j_{n}^{\prime}
\end{array}
$$

where $\left(j_{i}, j_{i}^{\prime}, \ldots\right)$ is the conjugate partition to $\left(k_{i}, k_{i}^{\prime}, \ldots\right)$.

The resulting array of $j$ 's is always a column-strict plane partition of shape $\left(k_{1}, k_{2}, \ldots, k_{n}\right)$ and largest part $n$, as defined in $\left[7, \S_{1}\right]$. The sum of the $j$ 's is equal to the sum of the $k$ 's. This correspondence between the array of $k^{\prime}$ 's (complete branchings with first row $k_{1}, k_{2}, \ldots, k_{n}$ and sum $m$ ) and $j$ 's (column-strict plane partitions of shape $\lambda=\left(k_{1}, k_{2}, \ldots, k_{n}\right)$ with largest part $n$ and sum $m)$ is easily seen to be a bijection. Hence $P_{n}(m)$ is equal to the number of column-strict plane partitions of shape $\lambda$ with largest part $n$ and sum $m$. In this context, the generating function (3) has been determined (implicitly) by D. E. Littlewood [5, p. 124, Theorem I] and more explicitly in [7, Theorem 15.3]. The result may be stated as follows.

Theorem 1. We have

$$
N(x)=x^{a} \prod \frac{(\mathbf{n}+\mathbf{j}-\mathbf{i})}{\left(\mathbf{h}_{\mathbf{i j}}\right)},
$$

where $(\mathrm{d})=1-x^{d}, a=\Sigma i k_{i}$, and $h_{i j}=k_{i}+\bar{k}_{j}-i-j+1$. Here $\left(\bar{k}_{1}, \bar{k}_{2}, \ldots\right)$ 
is the conjugate partition to $\left(k_{1}, k_{2}, \ldots\right)$, and the product is over all $k_{1}+$ $k_{2}+\cdots+k_{n}$ pairs $(i, j)$ such that $k_{i}>0$ and $\bar{k}_{j}>0$.

Some elementary manipulation shows that the right-hand side of (4) can be written in the form

$$
N(x)=\prod_{1 \leq i<j \leq n}\left(x^{k_{j}}-x^{k_{i}+j-i}\right) /(1) !(2) ! \cdots(\mathbf{n}-1) !
$$

where $(i) !=(1)(2) \ldots(i)$. If we put $x=1$ in (5) we obtain Gelbart's result (1). In the context of plane partitions this result was first given by MacMahon [6].

We shall give a brief indication of how (5) can be proved directly. Clearly one has

$$
N\left(k_{1}, \ldots, k_{n+1} ; x\right)=\sum x^{k_{1}^{\prime}+\cdots+k_{n}^{\prime}} N\left(k_{1}^{\prime}, \ldots, k_{n}^{\prime} ; x\right),
$$

where the summation is over all $k_{1}^{\prime}, \ldots, k_{n}^{\prime}$ such that $k_{1} \geq k_{1}^{\prime} \geq k_{2} \geq \cdots \geq$ $k_{n}^{\prime} \geq k_{n+1}$. But the $n \times n$ determinant

$$
D\left(k_{1}, \ldots, k_{n} ; x\right)=(-1)^{\left(\frac{n}{2}\right)} x^{n^{3}-n}\left|\left(\begin{array}{c}
\mathbf{k}_{\mathrm{j}}-\mathbf{j}-\mathbf{l} \\
\mathbf{i}-1
\end{array}\right)\right| .
$$

where $\left(\begin{array}{l}\mathbf{i} \\ \mathbf{j}\end{array}\right)=(\mathbf{i}) ! /(\mathbf{j}) !(\mathbf{i}-\mathbf{j})$ !, also satisfies the recursion (6) and equals $N\left(k_{1}, \ldots, k_{n} ; x\right)$ in the case $n=1$. It follows that $N\left(k_{1}, \ldots, k_{n} ; x\right)=$ $D\left(k_{1}, \ldots, k_{n} ; x\right)$. The determinant in (7) may be evaluated by Vandermonde's theorem or otherwise, yielding (5).

We remark that an evaluation of a determinant equivalent to (7) appears in [1]. See also $[7, \$ 15]$ for a direct proof that this determinant (actually a related but equivalent determinant) is given by (4). When $x=1$, the determinant (7) was first evaluated by Frobenius [2].

It follows from (5) that $N(x)=x^{A} N(1 / x)$, where $A=(n+1) \sum_{i=1}^{n} k_{i}$. From this we obtain the symmetry relation $P_{n}(m)=P_{n}(A-m)$. It would be interesting to know, whether for fixed $n, k_{1}, \ldots, k_{n}$, the sequence $\left\{P_{n}(m)\right\}$ is unimodal, that is, whether there exists a $C$ such that $P_{n}(m-1) \leq P_{n}(m)$ if $m \leq C$ and $P_{n}(m) \geq P_{n}(m+1)$ if $m \geq C$.

\section{REFERENCES}

1. L. Carlitz, Some determinants of q-binomial coefficients, J. Reine Angew. Math. 226 (1967), 216-220. MR 37 \#2773.

2. G. Frobenius, Über die charaktere der symmetrischen Gruppe, S.-B. Königl. Preuss. Akad. Wiss. Berlin, 1900, 516-534.

3. S. Gelbart, Problem 5834, Amer. Math. Monthly 79 (1972), 523; solution, ibid. 80 (1973), 819-820.

4. G. H. Hardy and E. M. Wright, An introduction to the theory of numbers, 4th ed., Oxford Univ. Press, London, 1960. 
5. D. E. Littlewood, The theory of group characters and matrix representations of groups, Oxford Univ. Press, New York, 1940. MR 2, 3.

6. P. A. MacMahon, Memoir on the theory of the partitions of numbers. IV, Philos. Trans. Roy. Soc. A 209 (1909), 153-175.

7. R. P. Stanley, Theory and application of plane partitions. I, II, Studies in Appl. Math. 50 (1971), 167-188, 259-279. MR 48 \#3754.

DEPARTMENT OF MATHEMATICS, DUKE UNIVERSITY, DURHAM, NORTH CAROLINA 27706

DEPARTMENT OF MATHEMATICS, MASSACHUSETTS INSTITUTE OF TECHNOLOGY, CAMBRIDGE, MASSACHUSETTS 02139 